\title{
An experience with COVID-19 positive pregnant patients at a tertiary care center
}

\author{
Anshu Baser, Akriti Gupta, Sunil Sharma*, Sushil Kumar
}

Department of Obstetrics and Gynaecology, MGM Medical College and Hospital, Navi Mumbai, Maharashtra, India

Received: 12 January 2021

Revised: 15 February 2021

Accepted: 16 February 2021

\author{
*Correspondence: \\ Dr. Sunil Sharma, \\ E-mail: sunilsharma2860@yahoo.com
}

Copyright: (c) the author(s), publisher and licensee Medip Academy. This is an open-access article distributed under the terms of the Creative Commons Attribution Non-Commercial License, which permits unrestricted non-commercial use, distribution, and reproduction in any medium, provided the original work is properly cited.

\begin{abstract}
Background: The Covid-19 pandemic is a global phenomenon. The lockdown imposed by the central and state governments, has also led to restriction of movement of patients thus reducing their access to antenatal care. Our hospital was officially declared as a Covid-19 centre as per government directives. The hospital has been managing Covid-19 positive patients as per guidelines. We here present the data of management of COVID-19 positive pregnant patients.

Methods: A retrospective study. All COVID positive patients admitted to the obstetric unit of the hospital from 23 March 2020 to November 2020 were analysed. A total of 96 COVID positive patients were analysed. The patients were analysed for antenatal complications, complications during delivery, mode of delivery and neonatal complications.

Results: There was only one patient with major complications associated with COVID. Most of the patients were either asymptomatic or had mild symptoms. There was only one maternal mortality. 23 out of the 89 antenatal cases required emergency caesarean section. The caesarean section rate was higher in COVID positive patients though the caesarean sections were done for obstetric indications only. Three neonates tested positive for the virus. 2 of the neonate required NICU admission.

Conclusions: COVID-19 is a completely unprecedented situation. The aim of this study was to demonstrate that the effect of COVID-19 on pregnant women is minimal. There were only 2 patients with major complications. The COVID positive pregnant patients can be managed effectively with proper personal protective equipment and adherence to guidelines.
\end{abstract}

Keywords: Antenatal care during lock down, Maternal mortality during Covid-19 lock down, Obstetrics during 'Covid-19' pandemic, Pregnancy in 'COVID-19' positive patients

\section{INTRODUCTION}

The Covid-19 pandemic is an unprecedented situation. Adequate studies are not yet available on ideal obstetric management during this situation. There are views and reviews but no clear-cut answers. ${ }^{1}$ There is fear all around. Patients are scared that they may develop complications and their fetus may not be safe. The Care takers like obstetricians, resident doctors, nursing and paramedical staff are also worried about their safety and the safety of their family. Some of the hospitals decline to take these patients and others do not find vacant bed to accommodate them. At this juncture our hospital was officially declared as a Covid-19 Centre by the government. We being a tertiary care centre have been managing both Covid-19 positive and negative cases. This is a preliminary study and our aim here is to present the details of obstetric management of Covid-19 cases as 
it was done in at a tertiary care centre. Also to emphasize Covid-19 infection in pregnant women is not associated with an increase in any major complications.

\section{METHODS}

A retrospective analysis was carried out at MGM Medical College and Hospital a tertiary care centre in Navi Mumbai. Data was collected from records maintained at the hospital. All data was analysed using SPSS software version 21. All the Covid positive patients admitted to the obstetric unit of the hospital from 23 March 2020 to November 2020 were analysed. There was no inclusion or exclusion criteria. The patients were analysed on the basis of antenatal complications, fetal loss, obstructed labour or caesarean section performed. The maternal morbidity and mortality was also recorded. Booked and unbooked cases were also analysed.

The Covid-19 positive patients were managed as per guidelines issued by the government authority and FOGSI.2 The entire staff wore personal protective equipment including N-95 masks during delivery. Patients were monitored intra partum for pulse rate, blood pressure, urine output and oxygen saturation was maintained above $94 \%$ at all times. The infants of Covid19 positive mothers were kept separately and not breast fed till 15 days post-delivery. The mothers were advised to express breast milk during the separation period.

The patient's underwent LSCS for obstetric indications unrelated to their COVID-19 positive status.

\section{RESULTS}

The data from 95 Covid positive patients were analysed (Table-1). The mean age of the patients was 24.5 years. All delivered patients were in between the age group of 18-30yrs (low risk group). Maximum patient belonged to middle or lower socioeconomic group (89\%). Out of these $52.7 \%$ were primiparas and rest were multiparas. Maximum of the multiparas $(60.5 \%)$ were previous sections evacuated from elsewhere due to positive COVID-19 report. $70 \%$ cases overall were referred from outside due to COVID positive status of the patient.

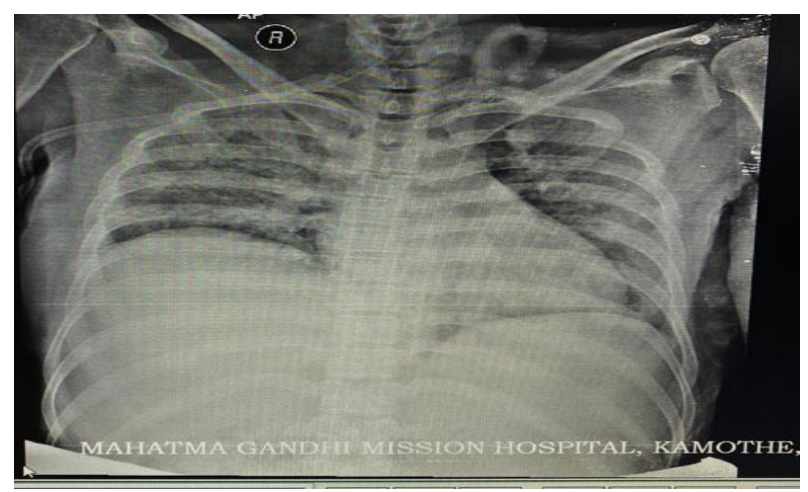

Figure 1: X-ray demonstrating bilateral infiltrates.
Among them 89 were antenatal cases and 6 were postnatal. Only 50 out of the 89 antenatal cases have delivered with us. The rest were discharged after 15 days and were lost to follow up. Most of the cases were asymptomatic or had minimal symptoms. (Figure 2) One antenatal case developed severe respiratory complications and required HDU management. 33 out of 50 patients who have been delivered so far underwent caesarean section due to obstetric indications. (Figure 3) 17 patients delivered normally. One of the patient who underwent normal delivery required intensive care management and was a subsequent maternal mortality.

Table 1: General statistics of pregnant Covid-19 positive patients.

\begin{tabular}{|l|l|}
\hline Total patients & $\mathbf{9 5}$ \\
\hline Antenatal cases & 89 \\
\hline Postnatal cases & 6 \\
\hline Asymptomatic cases & 20 \\
\hline Patients with mild symptoms & 6 \\
\hline Patient with major symptoms & 2 \\
\hline Mortality & 1 \\
\hline Patients delivered & 50 \\
\hline Emergency Caesarean & 23 \\
\hline Elective caesarean & 10 \\
\hline Vaginal delivery & 17 \\
\hline
\end{tabular}

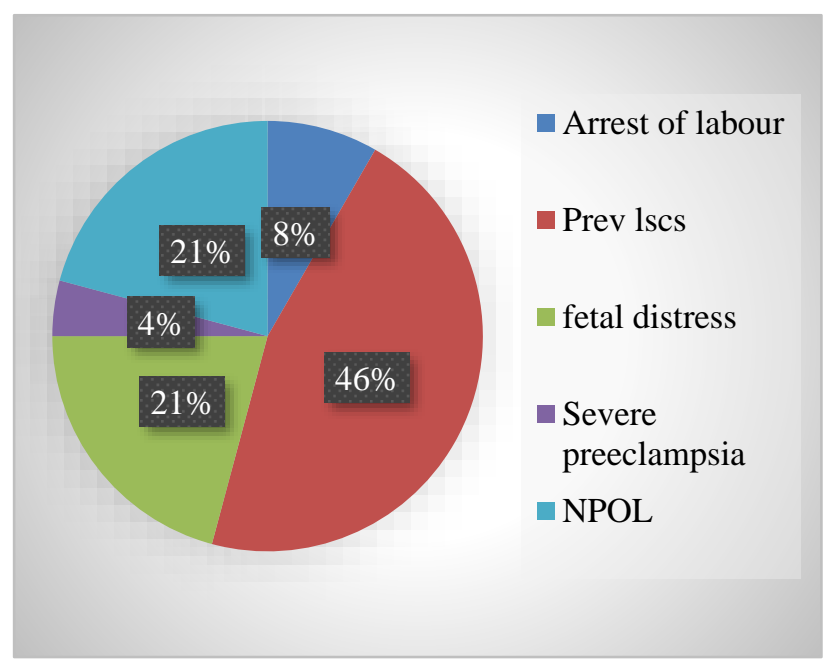

Figure 2: Indications for LSCS in COVID positive patients.

One of the antenatal patients who developed severe respiratory complications was a 32 weeker multigravida previous (vaginal delivery). Patient was admitted with breathlessness and was maintain saturation $85 \%$ on 15 litres of oxygen. Patient was given betamethasone $12 \mathrm{mg}$ 2 doses 24 hours apart. X-ray chest was done which showed bilateral infiltrates (Figure 1). Patient was given Tab Azithromycin od for 10 days, Inj. Remdesivir iv 5days along with heparin and corticosteroids. Urgent delivery was not planned for the patient as an obstetric ultrasound and doppler showed normal findings. Covid 
positive status is not an indication in its self to terminate the pregnancy. ${ }^{2}$ Patient gradually started improving and over the course of 20 days her oxygen requirement reduced and she was slowly weaned off oxygen. Patient was discharged at 34 weeks. She has not followed up since. Therefore the effects of antiviral therapy on the fetus are yet to be determined.

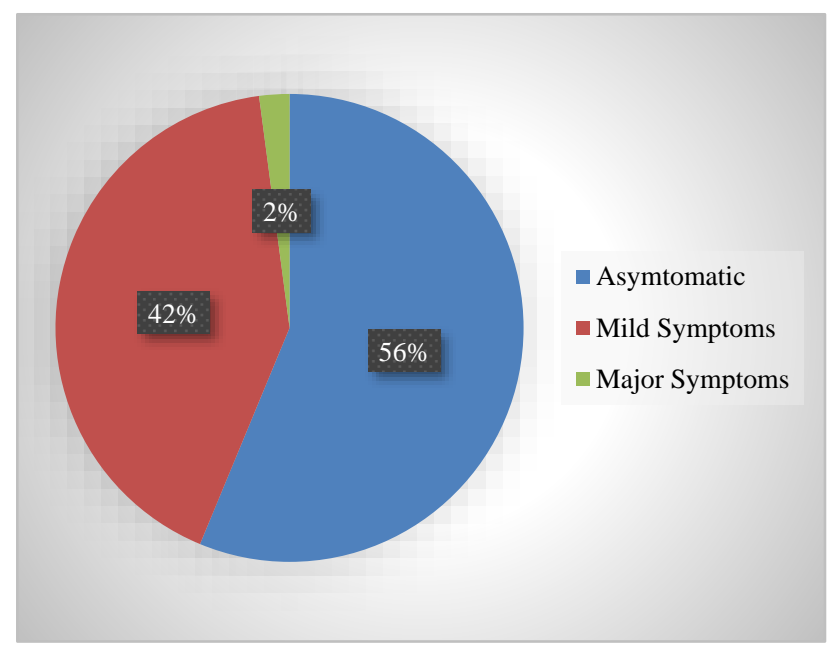

Figure 3: Symptomatology of Covid positive patients.

One of the delivered patients required intensive care management and ended in fatality. The patient had undiagnosed severe anaemia $\mathrm{Hb}-5 \mathrm{gm}$. She developed post-partum hemorrhage. She was transfused with 2 pint packed cells and 4 FFP. Patient was not maintaining bloop pressure and was started on ionotropic support. Patient was diagnosed with peripartum cardiomyopathy with and ejection fraction of only $20 \%$. Patient's SpO2 on room air was $85 \%$ and she was shifted to NIV support. Patient had ground glass opacification on CT with pleural effusion with pulmonary edema and dilated cardiomyopathy. Patient's serum creatinine was rising and required hemodialysis. Patient was not maintain vital parameters despite all supports, and could not be revived despite best efforts.

The was however no neonatal mortality. Two of the neonates required NICU admission one due to Meconium aspiration and the other due to very low birth weight (780 gm).

None of the postnatal patients had any major complications.

\section{DISCUSSION}

The Covid-19 pandemic will be recorded as an unparalleled event in human history. It has led to immense human suffering both in terms of physical illness as well as loss of earnings. Covid-19 virus is a novel virus. The genome of the virus has been sequenced and the vaccine will be perhaps the fastest developed vaccine in human history. ${ }^{3}$ The COVID-19 is an RNA virus which tends to mutate more commonly than the DNA viruses. These mutations lie on the surface of the protein, which makes COVID-19 more superior than other previous strains by inducing its sustainability leaving the immune system in a blind spot and therefore an effective vaccine has not yet surfaced. ${ }^{4}$ The course of the disease is more virulent in the very young or very old age group. ${ }^{5}$ Fortunately pregnant women do not fall in this group. It affects mainly the respiratory system which, as such, is slightly compromised during advanced pregnancy. The gold standard for the diagnosis of Covid19 infection is positive nasopharyngeal swab by RTPCR. ${ }^{6}$ Chest X-Ray and CT Chest also add to the diagnosis. $^{7}$

\section{Principal findings}

Most patients in our study were asymptomatic with a history of contact with positive cases or had mild symptoms such as cough and anosmia. As far as obstetrics is concerned, the effect of Covid-19 on pregnant women was minimal in our study and it is in conformity with other studies. ${ }^{8}$ Among the patients admitted in view of Covid-19 positive status there was no increase in rate of complications. Similar results are also shown in other studies. ${ }^{9}$ We did not however find an increased rate of preterm deliveries in Covid-19 cases as opposed to various studies. ${ }^{10}$ Only two of our Covid positive patients required ICU admission.

Antiviral therapy with Remdesivir is currently not recommended in the FOGSI GCPR guidelines, however in light of the maternal condition we administered these with taking proper consent of the patient and relatives. ${ }^{2}$

Fetal effects will require further study. The overall caesarean section rate in our tertiary care institution is $28 \%$.

The incidence of caesarean section was however higher in our study. Covid-19 positive patients had $66 \%$ Caesarean section rate compared to $34 \%$ rate of vaginal delivery (Table 1).

However the patients were taken for LSCS for obstetric indications not due to Covid-19 positive status as evident in Figure 3.

The mothers and infants were kept separately till the patients tested negative. Two of the neonates required NICU admission. Two out of the 50 delivered neonate came positive and none had any congenital anomaly. The neonate was in no direct contact with the mother however tested positive 36 hours after birth. Although being a tertiary care medical centre there is a chance of cross contamination. The possibility of vertical transmission was controversial and literature regarding the same is scarce..$^{10,11}$ 
There is now however evidence available of vertical transmission, the virus being detected in various body fluids. Fetal effects appear to be minimal however long term studies are further required. ${ }^{2}$ Vertical Transmission cannot be ruled out here. ${ }^{12}$

Limitations of the study: The current study is limited by cost constraints as no financial aid was obtained for this study and most of our patients could not afford extensive investigations and therefore statistical analysis of laboratory parameters could not be carried out. Also being a tertiary care centre many patients were lost to follow up and long term effects could therefore not be studied.

Due to the possibility of cross contamination vertical transmission cannot be stated definitely however the possibility cannot be ruled out as well.

\section{Clinical implications}

Increase caesarean section rate could possibly due to the obstetrician's low threshold and high alertness in COVID-19 patients. Pregnant women though at risk do not seem to have any major complications as demonstrated in our study. There were also no major neonatal complications however we do recommend availability of a NICU setting while delivering COVID19 patients.

\section{Research Implications}

Most diseases require decades of research and study before effective therapy and vaccines are available. Developing literature requires time.

Due to the widespread morbidity and mortality associated with the COVID-19 pandemic all of this has been fast forwarded. This study aims to add to the relatively scarce data that we have available worldwide in an attempt to better understand and treat this disease.

Vertical transmission in COVID-19 patients is still under surveillance, a larger population study is required to determine exact incidence. Additional studies are required to comment on the higher caesarean section rate in COVID-19 patients that was found in our study. Management of patients with respiratory complications also requires further study.

\section{CONCLUSION}

The COVID-19 situation has increased the burden on the health care centre.

However there was no increase in obstetric complications in Covid-19 positive patients as compared to patients who are Covid-19 negative. Only 2 of our Covid positive, obstetrics patients, needed ICU admission and two of the neonate required NICU admission. Only three of the 50 neonate delivered were found to be Covid-19 positive.

To sum up, the study shows that Covid-19 infected patients can be managed effectively if proper personal protective equipment is used and guidelines issued are followed, without a significant increase in complication rate.

Funding: No funding sources

Conflict of interest: None declared

Ethical approval: The study was approved by the Institutional Ethics Committee

\section{REFERENCES}

1. Kumar S. COVID-19 pandemic: Only views, counter views, and reviews, no clear-cut answers yet. MGM J Med Sci. 2020;7:1-4.

2. Gandhi A, Ganatra A, Tank P. Good Clinical Practice recommendation on PREGNANCY WITH COVID-19Infection. FOGSI GCPR. 2020;1-55.

3. Kim J, Chung Y, Jo H, Lee N, Kim M, Woo S. Identification of Coronavirus Isolated from a Patient in Korea with COVID-19. Osong Public Health and Research Perspectives. 2020;11(1):3-7.

4. Abdelmageed M, Abdelmoneim A, Mustafa M. Design of a Multiepitope-Based Peptide Vaccine against the E Protein of Human COVID-19: An Immunoinformatics Approach. BioMed Research International. 2020;2020:1-12.

5. Zhou F, Yu T, Du R, Fan G, Liu Y, Liu Z. Clinical course and risk factors for mortality of adult inpatients with COVID-19 in Wuhan, China: a retrospective cohort study. The Lancet. 2020;395(10229):1054-62.

6. Goudouris E. Laboratory diagnosis of COVID-19. J de Pediatria. 2020.

7. Ai T, Yang Z, Hou H, Zhan C, Chen C, Lv W, et al. Correlation of Chest CT and RT-PCR Testing for Coronavirus Disease 2019 (COVID-19) in China: A Report of 1014 Cases. Radiology. 2020;296(2):E32E40.

8. Chen L. Clinical characteristics of pregnant women with Covid-19 in Wuhan, China. N Engl J Med 2020.

9. Qiancheng X, Jian S, Lingling P, Lei H, Xiaogan J, Weihua L, et al. Coronavirus disease 2019 in pregnancy. International $\mathrm{J}$ Infectious Dis. 2020;95:376-83.

10. Smith V, Seo D, Warty R, Payne O, Salih M, Chin K et al. Maternal and neonatal outcomes associated with COVID-19 infection: A systematic review. PLOS ONE. 2020;15(6):e0234187.

11. Chawla D, Chirla D, Dalwai S, Deorari A, Ganatra A, Gandhi A et al. Perinatal-Neonatal Management of COVID-19 Infection-Guidelines of the Federation of Obstetric and Gynaecological Societies of India (FOGSI), National Neonatology Forum of India 
(NNF), and Indian Academy of Pediatrics (IAP). Indian Pediatr. 2020;57(6):536-48.

12. Patanè L, Morotti D, Giunta $M$, Sigismondi C, Piccoli M, Frigerio L, et al. Vertical transmission of coronavirus disease 2019: severe acute respiratory syndrome coronavirus 2 RNA on the fetal side of the placenta in pregnancies with coronavirus disease 2019-positive mothers and neonates at birth. American J Obstetr Gynecol MFM. 2020;2(3):100145.

Cite this article as: Baser A, Gupta A, Sharma S, Kumar S. An experience with COVID-19 positive pregnant patients at a tertiary care center. Int J Reprod Contracept Obstet Gynecol 2021;10:1463-7. 